\title{
Neutrino telescopes' sensitivity to dark matter
}

\author{
Ivone F. M. Albuquerque* \\ Space Science Laboratory and Astronomy Department, University of California, Berkeley, California 94720 \\ Jodi Lamoureux ${ }^{\dagger}$ \\ National Energy Research Scientific Computing Center, Lawrence Berkeley National Laboratory, Berkeley, California 94720 \\ George F. Smoot \\ Lawrence Berkeley National Laboratory, Space Sciences Laboratory and Department of Physics, University of California, Berkeley, \\ California 94720 \\ (Received 15 May 2002; published 18 December 2002)
}

\begin{abstract}
The nature of the dark matter of the Universe is yet unknown and most likely is connected with new physics. The search for its composition is underway through direct and indirect detection. Fundamental physical aspects such as energy threshold, geometry and location are taken into account to investigate proposed neutrino telescopes of $\mathrm{km}^{3}$ volume sensitivities to dark matter. These sensitivities are just sufficient to test a few weakly interacting massive particle scenarios. Telescopes of $\mathrm{km}^{3}$ volume, such as IceCube, can definitely discover or exclude superheavy $\left(\mathrm{M}>10^{10} \mathrm{GeV}\right)$ strong interacting massive particles (simpzillas). Smaller neutrino telescopes such as ANTARES, AMANDA-II and NESTOR can probe a large region of simpzilla parameter space.
\end{abstract}

DOI: 10.1103/PhysRevD.66.125006

\section{INTRODUCTION}

Most of the Universe's matter is non-luminous. There are many predictions for its composition but the nature of dark matter is yet unknown. The possibility of accounting for this matter within the standard model of particle physics is small and is constrained both by particle physics and by cosmology. Discovery of the dark matter composition will probably reveal new physics.

Many models based in physics beyond the standard model predict the existence of new particles as a solution to the dark matter problem. These new particles can be detected directly through interactions with nuclei recoil or through secondary products of their annihilation. The decay of particles produced in the annihilation or decay of these new particles generates a flux of high energy neutrinos. The direct and the secondary neutrino signatures are complementary to each other. Detection - or lack of detection - of the secondary neutrinos is therefore important for understanding the nature of dark matter as well as for uncovering the new physics.

A leading dark matter candidate is the weakly interacting massive particle (WIMP). A thermal stable relic from the early Universe, its abundance is inversely proportional to its thermal averaged annihilation cross section $\langle\sigma v\rangle$. If this cross section is estimated from the weak-scale interactions, the resulting abundance is close to the observed energy density of dark matter [1]. This coincidence makes WIMPs a strong candidate for the non-luminous matter.

However, the WIMP is a broad category and its identity and character are yet unknown. The most investigated candidate is the neutralino, proposed as the lightest supersym-

\footnotetext{
*Electronic mail: IFAlbuquerque@lbl.gov

†Electronic mail: JILamoureux@lbl.gov

${ }^{\ddagger}$ Electronic mail: GFSmoot@lbl.gov
}

PACS number(s): 13.15.+g, 95.35.+d, 98.80.Cq

metric particle (LSP) within the minimal supersymmetric standard model (MSSM). If neutralinos exist and compose the dark matter of the Universe, they should be captured in the Sun and in the Earth. Once captured they should concentrate near the center and annihilate with each other into particles which would produce high energy neutrinos.

Another dark matter candidate is a strongly interacting massive particle (SIMPs). Capture and annihilation of superheavy (mass above $\sim 10^{10} \mathrm{GeV}$ ) SIMPs (simpzillas) in the Sun or in the Earth will also produce secondary high energy neutrinos [2].

In order to realistically understand neutrino telescopes' sensitivity to dark matter, we first summarize predictions of the muon rate arriving at neutrino telescopes from WIMPs. A discussion follows on the feasibility of detecting muons produced by these neutrinos once the detector location and threshold is taken into account. Although based in rates predicted by others, our discussion is new and intends to make a more realistic prediction of WIMP detection in neutrino telescopes'.

We then review the estimate for the flux of charged current induced taus from neutrinos produced in the annihilation of simpzillas in the Sun [2]. From there we estimate the detection rate of current or proposed detectors such as AMANDA-II, ANTARES, IceCube and NESTOR, taking the background into account. We also consider a km-scale detector in the Mediterranean Sea which would correspond to a larger version of ANTARES or NESTOR. In estimating the detection rate we determine the optimum detector energy threshold for indirect detection of simpzillas.

We show that when the fundamental physical aspects of neutrino telescopes are considered discovery of dark matter is assured, if it is composed of strongly interacting superheavy particles. The sensitivity of proposed $\mathrm{km}^{3}$ volume detectors is only able to probe a few WIMP models in a currently very large phase space of models. 

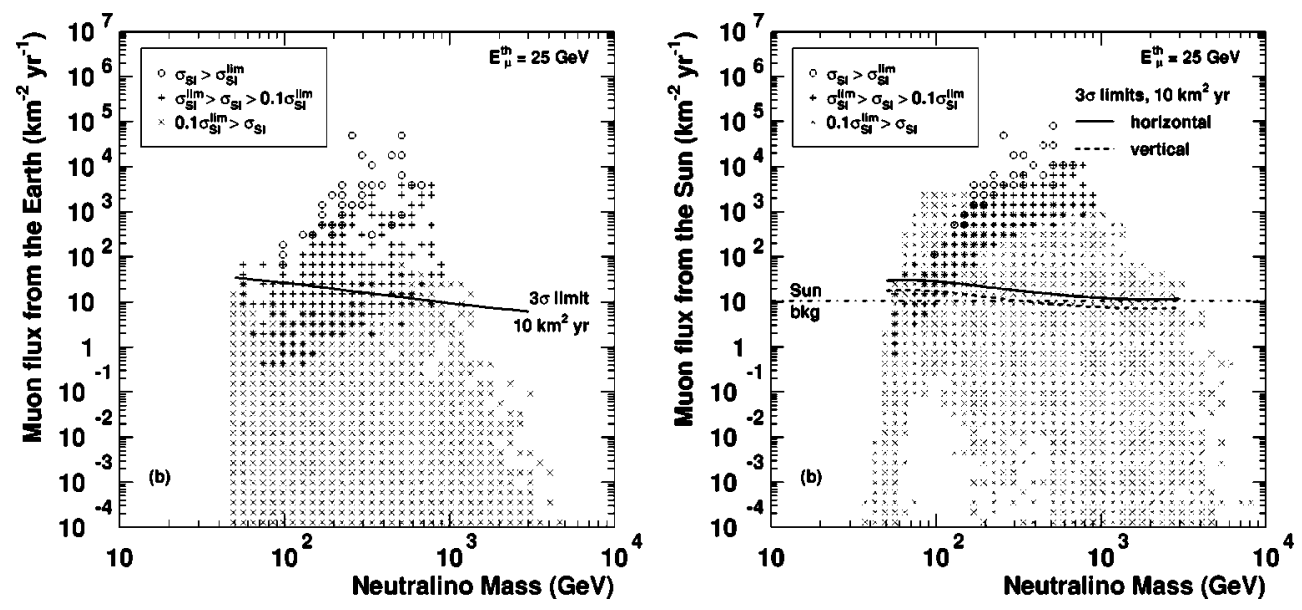

FIG. 1. The muon fluxes versus the neutralino mass for annihilation in the Earth and in the Sun obtained by Bergstrom, Edsjo and Gondolo [8]. The muon energy threshold is $25 \mathrm{GeV}$. The horizontal line is a $3 \sigma$ limit to be reached with an exposure of $10 \mathrm{~km}^{2} \mathrm{yr}$. The rate shown does not include detector geometry and instrumental effects. MSSM models excluded by direct detection experiments are shown with circles and the ones that will be probed by future direct detection experiments with about a factor of 10 improvement in sensitivity are shown with a plus sign. (The figure was extracted from [8].)

\section{WIMPS-NEUTRALINO}

As discussed above, the WIMP is a promising dark matter candidate. Within the WIMP category, neutralinos-the lightest supersymmetric particle (LSP) — is the most investigated. The search for a neutralino signature is a search not only for dark matter but also a test for the supersymmetric extension of the standard model [1].

There are mainly two ways to detect WIMPs. One is a direct detection through the scattering of WIMPs from nuclei. If the halo of our galaxy consists of WIMPs, they will pass through the Earth. Although their cross section with ordinary matter is weak and the interactions rare, it is still possible to measure the small energy liberated from the nuclear recoil due to a WIMP-nucleus scattering. CDMS [3] and GENIUS [4] are examples of experiments that will directly search for WIMP-nucleus interactions.

Indirect detection involves looking for annihilation or interaction products that propagate through space to the detector. Several simulations of this process have been done [5-8] estimating the rate of charged current induced muons arriving in neutrino telescopes. These simulations scan through MSSM parameters assuming that the neutralino is the WIMP. An analytical approach has also been developed [9] and allows for a better understanding of the physical processes such as the capture and annihilation of WIMPs and consequent particle production.

\section{A. High energy neutrino signature from neutralinos}

When WIMPs from the halo pass through the Sun or Earth material, they may lose energy by elastic scattering with nuclei. If their velocities are reduced below the escape velocity, they are trapped in the Sun or Earth $[10,11]$ and further scattering concentrates them together in the center. They annihilate with their anti-particles and produce leptons, quarks and, if heavy enough, gauge bosons, Higgs bosons and top quarks [9]. Most of these products will not escape the Sun or the Earth. However, neutrinos produced from the decay of these particles will escape [9].

Muon neutrinos coming from the Sun or going through the Earth will occasionally interact with a nucleus and produce muons in a charged current interaction. They will also have their energy degraded due to a neutral current interaction. The cross sections for these processes [12] are small but the combination of the density and the distance from the center of the Earth or from the Sun is enough to allow for significant muon production. After estimating the muon rate near the detector, one has to account for the effective size of the detector in order to predict the muon detection rate [13].

One has also to account for the background. For neutrinos coming from the Sun the primary background is neutrino production from cosmic ray interactions in the solar and terrestrial atmospheres. The indirect neutralino detection rate in neutrino telescopes from simulations of neutralino annihilation has been estimated by Bergstrom, Edsjo and Gondolo (BEG) $[7,8]$.

Their analysis includes the background from cosmic ray interactions in the Earth and Sun atmospheres. In order to reduce this background, they use events within an angular cone of the center of the Earth or of the Sun. They use the method described in [6] where it is shown how the angular and/or energy resolution of the detector can improve the sensitivity of neutrino telescopes. However, as in general these telescopes have poor energy resolution, only the angular resolution is used to reduce the background.

The result from the (BEG) analysis [8] is shown in Fig. 1, indicating the estimated $3 \sigma$ limit for neutralino annihilation in the Earth and in the Sun obtained for an exposure of $10 \mathrm{~km}^{2}$ year and a $25 \mathrm{GeV}$ energy threshold. Also shown is the irreducible background from cosmic rays interacting in the Sun. A similar but reducible background exists for the Earth in atmospherically produced neutrinos.

BEG [8] also show the effect of the detector energy threshold in the muon detected rates. This is shown in Fig. 2. 

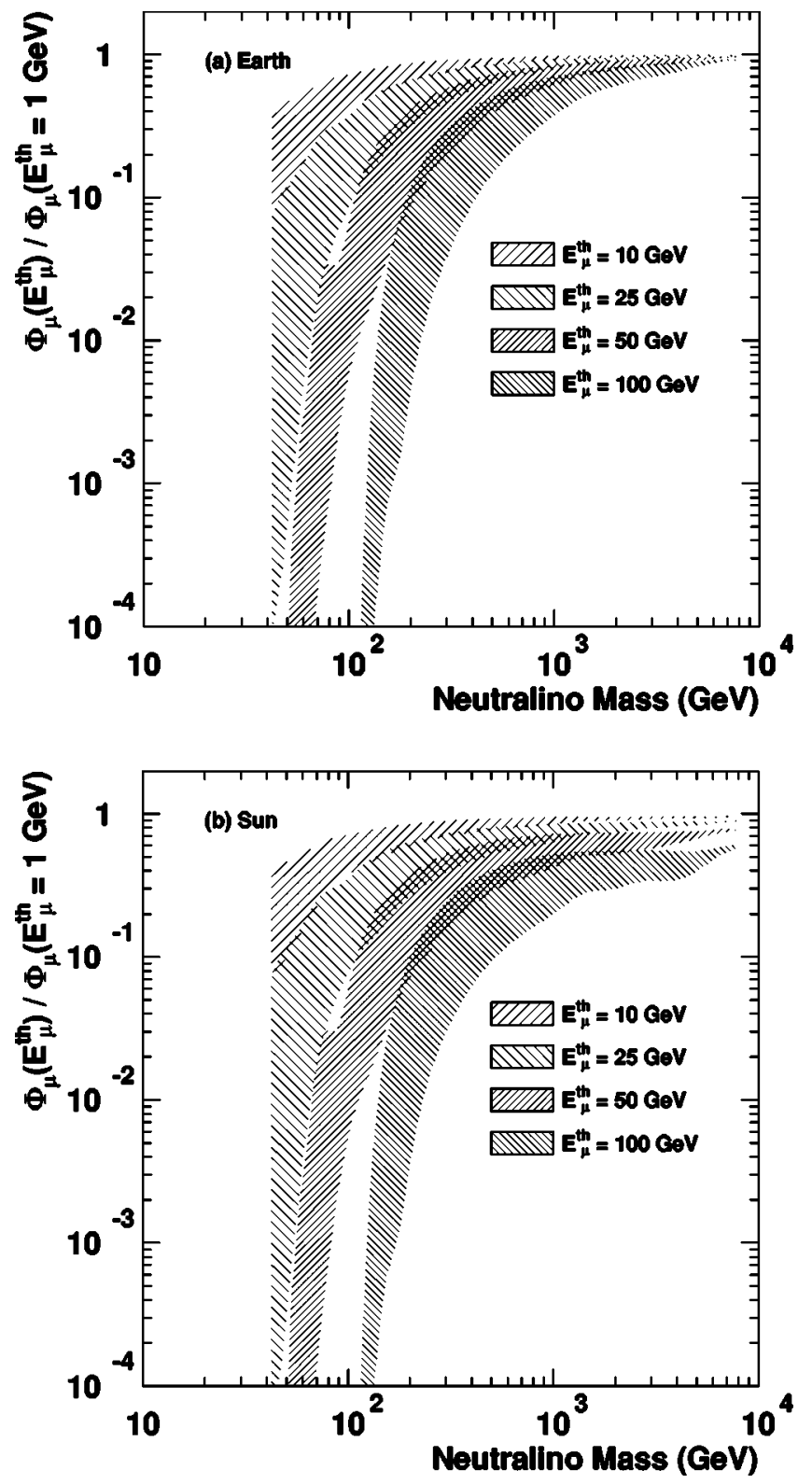

FIG. 2. The ratio of the muon fluxes with a threshold of $\mathrm{E}_{\mu}^{\text {th }}$ to those with a threshold of $1 \mathrm{GeV}$ in (a) the Earth and (b) the Sun versus the neutralino mass. For each given threshold, a band of allowed ratios is given. (The figure and caption were extracted from [8].)

Different energy thresholds are compared to a $1 \mathrm{GeV}$ threshold. The band represents different degrees of softness of the neutrino spectra for a given neutralino mass. It represents the fact that the annihilation branching fractions are unknown and the resulting neutrino energy spectra might vary depending on the number of each annihilation product. A $100 \mathrm{GeV}$ threshold would considerably reduce the signal rate.

The analysis [8] summarized here shows that the potential for detection of neutrinos from neutralino annihilation in the Earth is comparable with the estimated direct detection sensitivity whereas the detection from annihilation in the center of the Sun is more promising for neutrino telescopes. However, the muon rate will depend on the detector geometry, energy threshold, location and also on instrumental effects. In Sec. II C we will expand the analysis described above to include these effects.

\section{B. Neutrinos from constrained MSSM (CMSSM)}

Recently constrains from experimental results were used to select MSSM scenarios. These results include searches for sparticles, Higgs boson and $b \rightarrow s \gamma$ decay rate. The CMSSM parameter space also constrains the supersymmetric relic density to be within the $0.1<\Omega_{\chi} h^{2}<0.3$ range which is set by cosmological observations.

A set of parameters within the CMSSM has been chosen in order to probe this model in a more systematic way [14]. This set was used to select benchmark models that are representative of a particular set of parameters. Thirteen models were selected with this purpose.

Direct and indirect signatures of the benchmark models are estimated in [15]. The neutrino charged current induced muon flux from benchmark models is determined and their results are compared with the quoted sensitivity of neutrino telescopes. These are shown in Fig. 3.

A few representative models can be indirectly tested from neutralino annihilation in the Sun but not in the Earth. Two of these models (E and F) have exceptional flux. Both models predict neutralinos with significant Higgsino content which leads to annihilations into gauge bosons. Gauge bosons will produce hard neutrinos [9] and the muon rate will be enhanced.

IceCube sensitivity is enough to probe models $\mathrm{B}, \mathrm{E}$ and $\mathrm{F}$ shown in Fig. 3. Models I, G, C and L are on the borderline. However some models are below the detector energy threshold. We will consider this aspect in Sec. II C.

Gravity-mediated supersymmetry breaking in supergravity is also used [16] to constrain the MSSM [minimal supergravity (MSUGRA) model]. It allows for a wider range of the cosmological relic density $0.03<\Omega h^{2}<0.3$ and is defined by a 5 parameter constrained MSSM. The muon flux from neutralino annihilation in the Sun from a set of MSUGRA models is estimated as well as the direct detection rate. One problem with this analysis is the low detector energy threshold which is assumed to be $5 \mathrm{GeV}$. This will be discussed in Sec. II C. The conclusion of this analysis is that indirect detection of neutralinos assuming the MSUGRA constraints is beyond reach of present and future neutrino telescopes for annihilation in the center of the Earth. For annihilation in the Sun, the region where the neutralino has a larger Higgsino content (which increases the elastic scattering and neutralino annihilation cross sections) can be probed.

\section{Discussion}

Three scenarios for indirect detection of WIMPs were summarized in this section: a search for neutrino signature from a broad set of parameters within the MSSM, a search for CMSSM benchmarks and a search for models within MSUGRA. In all scenarios, models for WIMP annihilation in the Sun predicts a higher neutrino rate than annihilation in 

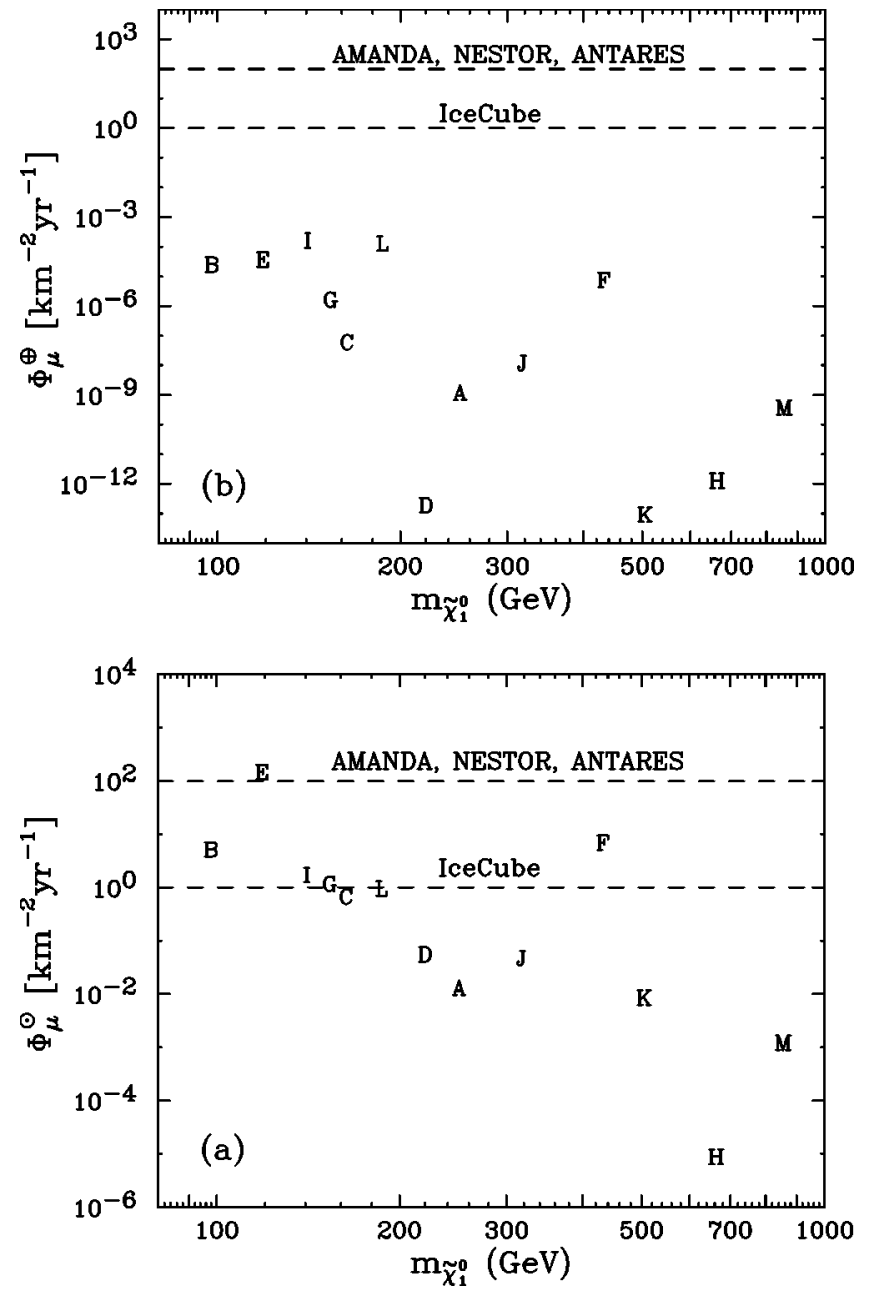

FIG. 3. CMSSM predictions for neutrino induced muon flux from neutralino annihilation in the Earth (top) and in the Sun (bottom). Each character represents a model within the CMSSM. Parameters of each set of model are given in [15]. Sensitivities for AMANDA II [17], NESTOR [18], ANTARES [19] and IceCube [20] are also shown. The figure was extracted from [15].

the Earth. We now discuss how the basic physical aspects of neutrino detectors such as geometry, location and threshold will affect these results.

An analysis of the fundamental physical aspects of instrument performance for neutrino telescopes is described in [13]. The basic physical characteristics of an ideal detector are taken into consideration. An ideal detector performance is translated to include characteristics of current and proposed detectors. The geometry and location of such detectors are taken into consideration.

The prediction [8] of MSSM scenarios to be tested by neutrino telescopes is shown in Figs. 1 and 2. These results depend strongly on a low energy threshold. Also the rates are determined for 10 years of $100 \%$ efficiency in a $\mathrm{km}^{2}$ incident area. If one includes the duty cycle of the detector in a year this will translate to a longer period in real time.

The best threshold to be achieved by a $\mathrm{km}^{2}$ incident area detector is set by the minimum ionizing muon energy loss of about 2.6 MeV/cm. A $25 \mathrm{GeV}$ threshold implies that a 125 meter track was observed. To determine the track length, a minimum of a few photomultiplier tubes (PMTs) have to be hit. Efficient coverage of a $\mathrm{km}^{3}$ with 5 hits per $125 \mathrm{~m}$ would then require a 64,000 channel detector. The largest proposed neutrino detector is IceCube with 5000 channels [21]. Spreading the channels over a larger area has the effect of raising the energy threshold well above $25 \mathrm{GeV}$. IceCube will consist of strings with $125 \mathrm{~m}$ string spacing and $16 \mathrm{~m}$ PMTs spacing on each string. Simulations of the energy threshold indicate $\mathrm{E}_{\mu}^{\text {thr }}>10 \mathrm{GeV}$ for vertical tracks and 200 $\mathrm{GeV}$ for horizontal tracks with something close to $0.7 \mathrm{~km}^{2}$ volume [21]. Since these predictions do not include background rejection of atmospheric muons, it is insightful to note that the AMANDA-II energy threshold for muons is 50 $\mathrm{GeV}[22,23]$ which translates into a neutralino threshold of $\mathrm{M}_{\chi} \gtrsim 100 \mathrm{GeV}$. The vertical spacing of PMTs in IceCube is similar to AMANDA leading us to conclude that a similar threshold may apply. However, as AMANDA and IceCube are located in the South Pole, the neutrinos coming from the Sun will be arriving at the detector almost horizontally. This implies that the energy threshold will be closer to $200 \mathrm{GeV}$ which translates into a higher neutralino threshold of $\mathrm{M}_{\chi}$ $\gtrsim 400 \mathrm{GeV}$. In this sense ANTARES is better located since neutrinos from the Sun enter the detector at all zenith angles. A $\mathrm{km}^{3}$ version of ANTARES would be able to perform an analysis as a function of the zenith angle.

High energy thresholds reduce the MSSM parameter space to be tested by neutrino telescopes. Figure 2 shows that the muon rate in the detector will be suppressed by about a factor of 10 around $100 \mathrm{GeV}$ and by a factor of 5 around $1000 \mathrm{GeV}$ when going from a $25 \mathrm{GeV}$ to a $100 \mathrm{GeV}$ threshold. In IceCube, limits from the Sun will be several orders of magnitude worse than shown in Fig. 1 below a neutralino mass of $150 \mathrm{GeV}$ and a few times worse elsewhere due to the detector energy threshold.

This together with the fact that for a $25 \mathrm{GeV}$ threshold the background from the Sun for neutralino masses around 100 $\mathrm{GeV}$ requires a few tens of $\mathrm{km}^{2}$ instead of a $10 \mathrm{~km}^{2}$ exposure due to the background from the solar corona [8] which lowers the possibility of indirect discovery of WIMPs in this mass region. At higher energies $(\sim 1 \mathrm{TeV})$ the rate will be suppressed by a factor of 5 due to the higher threshold and reduced flux.

The geometrical efficiency of big detectors like IceCube is close to unity taking into account muons that travel $300 \mathrm{~m}$ or more [13]. The primary low energy muon background comes from atmospheric muons and muon neutrinos produced in cosmic ray interactions in the atmosphere. Specifically, only up-going measurements are possible [4]. WIMPS from the Sun therefore, may only be detected at night when the Sun, is below the horizon. In the South Pole, there is night for 6 months when the Sun dips a maximum of $23.5^{\circ}$ below the horizon. At Mediterranean latitudes, the Sun is below the horizon each night. For both locations exposure times of 10 years will actually take 20 years of nightly observing.

It is also important to note that the direct and indirect detection will be complementary each to the other. Although a large fraction of the region to be probed by indirect detec- 
tion can also be probed by direct detection, an agreement among these two techniques is important.

From the 13 CMSSM benchmarks (see Sec. II B) [14] one is within the quoted IceCube sensitivity and above its energy threshold (model F). Four others (I,G,C and L) are on top of the quoted sensitivity which makes their detection borderline. Models B and E fall below the detector energy threshold. The others cannot be probed by IceCube. An expanded version of ANTARES may be able to probe models E and B. As ANTARES is located in the Mediterranean, neutrinos coming from the Sun enter the detector in all angles. For this reason the energy threshold for neutrinos coming from the Sun is lower than a detector in the South Pole with same PMT spacing.

For models within the MSUGRA framework (see Sec. II B) [24] the indirect detection is only possible for neutralino annihilation in the Sun for neutralinos with a large Higgsino content. However, it was determined for a detector energy threshold of $5 \mathrm{GeV}$ which is too low for a telescope such as IceCube. The results shown for ANTARES might be better represented since, as mentioned above, the ANTARES energy threshold is lower than IceCube, though $5 \mathrm{GeV}$ is still not representative of the average energy threshold.

We conclude this section by stating that the WIMP parameter space is very large, and detectors located in the South Pole (as the proposed IceCube experiment) or in the Mediterranean (as would be an expanded version of ANTARES or NESTOR) will probe only those few models with the largest neutrino fluxes. Models with lower fluxes can be probed if the energy thresholds are lowered and experimental live time is extended beyond 5 years ( 10 years of nightly observing). However, as will be shown in the section that follows, if dark matter is composed by superheavy strongly interacting particles their discovery will be guaranteed for the most natural scenarios.

\section{SIMPZILLAS}

It has been proposed by Chung, Kolb and Riotto [25] that the dark matter of the Universe might be composed of a supermassive (mass greater than $10^{10} \mathrm{GeV}$ ) stable particle that has never been in thermal equilibrium with the primordial plasma. In order not to be in thermal equilibrium the particle annihilation rate must be smaller than the expansion rate. Essentially the large mass prevents the particle from thermalizing. As it is not a thermal relic their abundance is not determined by their self-annihilation cross section but by their mass. These particles can be produced in many ways [25]. Among their production mechanisms are the decay of the inflaton field, gravitational at the end of inflation or through a broad resonance mechanism of preheating. The inflaton mass $\left(\sim 10^{12} \mathrm{GeV}\right)$ is an appropriate scale for their mass [25].

As the abundance of these superheavy particles depends on their mass and not in their interaction strength they might interact strongly or weakly with normal matter. If they are strongly interacting and constitute the dark matter of the Universe, they are captured in the Sun as well as in the Earth as shown in Albuquerque, Hui and Kolb (AHK) [2]. They lose energy while scattering with the Sun or Earth's matter and are trapped in there once their velocity is smaller than the escape velocity. The same happens with WIMPs but with one important difference: WIMPs interact weakly with normal matter and the optical depth of the Sun for these particles is much less than unity; whereas for simpzillas, which interact strongly, the optical depth is much greater than one. The capture and annihilation rate of Simpzillas in the Sun and in the Earth is estimated in [2]. The Sun is more efficient in simpzilla capture and therefore the annilhilation of these particles will produce a greater neutrino flux than the one from simpzilla annihilation in the Earth. For this reason, we consider the high energy neutrino signature from annihilations in the Sun.

As simpzillas are ultra-heavy particles with masses above $\sim 10^{10} \mathrm{GeV}$, their annihilation produces hadronic jets which include top quarks [2]. The neutrino rate from top decay in the core of the Sun is estimated as well as the neutrino emission rate from the Sun. As shown in AHK [2] the rate of muon and electron neutrinos are suppressed since once they convert into a lepton through a charged current interaction (CC) the lepton will lose most of its energy before decaying. The same is not true for tau neutrinos since the tau produced in the CC will immediately decay. As a consequence the tau neutrino emergent rate is about twice the muon and electron neutrino rates. However, if neutrino oscillations are taken into account, tau neutrinos might oscillate into muon neutrinos and vice versa. This effect will be discussed in Sec. IV C. Another enhancement of muon and electron neutrinos are due to taus decaying close to the edge of the Sun. The tau decays $18 \%$ of the time into $\nu_{\tau} \mu \nu_{\mu}$ and another $18 \%$ into $\nu_{\tau} e \nu_{e}$. We call these $\nu_{e}$ and $\nu_{\tau}$ secondary neutrinos and will take them into account when discussing oscillations.

In Sec. IV we estimate the sensitivity of neutrino telescopes to tau neutrinos from simpzilla annihilation in the Sun. We take the arriving flux of tau neutrinos at the Earth from the Sun as determined in Eq. (5.1) of [2]. This rate depends on the simpzilla mass $\left(M_{\chi}\right)$ and the simpzillanucleon cross section $\left(\sigma_{\chi-N}\right)$. Figure 4 shows the tau neutrino differential flux arriving at the Earth weighted by neutrino energy for different values for $M_{\chi}$ and $\sigma_{\chi-N}$. This flux will be enhanced by the anti-tau contribution. The allowed $M_{\chi}$ versus $\sigma_{\chi-N}$ space is shown in Fig. 7.

It is important to note that the results from [2] include only neutrinos produced from top decay. The hadronic jets produced in simpzilla annihilation also produce $B$ mesons which also decay into neutrinos of all flavors. They will therefore enhance the neutrino rate. The shape of the neutrino energy spectrum from $B$ decays should be the same as that for top decay. Also the flux shown in Fig. 4 does not include the anti-neutrinos.

The background for these events consists mainly of atmospheric neutrinos, solar neutrinos and atmospheric muons. The atmospheric muon background is huge for downgoing events but insignificant for the upgoing ones. As we will show in Sec. IV the upgoing event rate from simpzillas produced neutrinos is sufficiently large that one does not need to consider the downgoing ones.

In Sec. IV we determine the $\nu_{\tau}$ contained event rate in a 


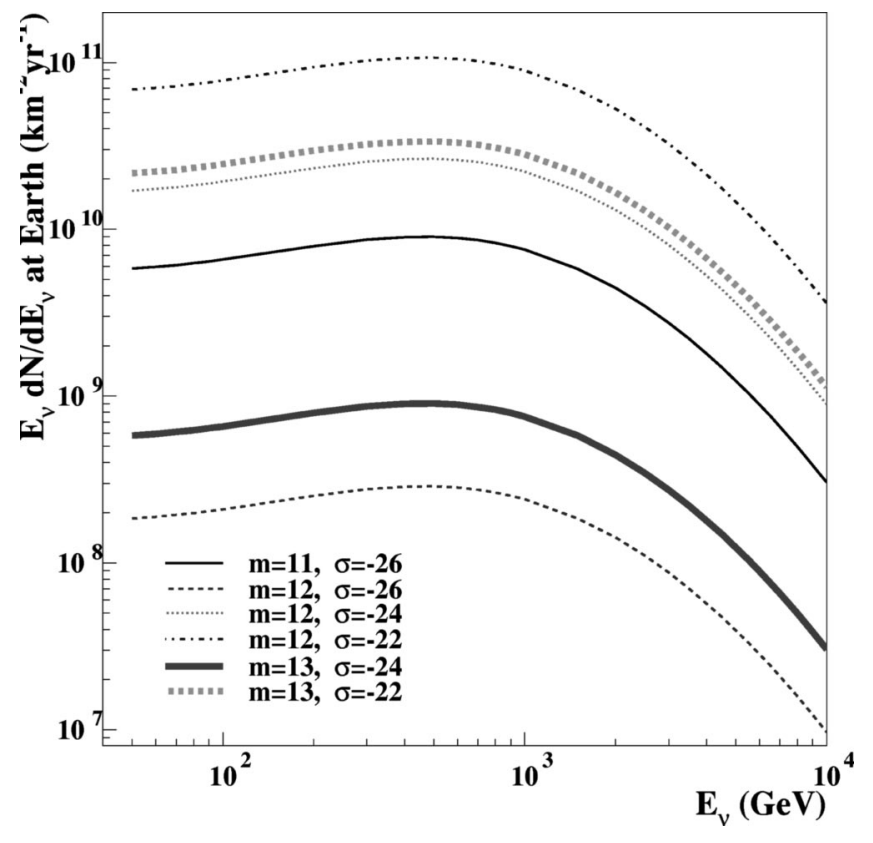

FIG. 4. Tau neutrino differential rate at the Earth weighted by neutrino energy from simpzilla annihilation in the Sun. Values assumed for $M_{\chi}$ and $\sigma_{\chi-N}$ are labeled as $10^{\mathrm{m}} \mathrm{GeV}$ and $10^{\sigma} \mathrm{cm}^{2}$, respectively. Anti-taus are not included.

perfect detector of $\mathrm{km}^{3}$ volume as well as the sensitivity of such a detector for neutrinos from simpzilla annihilation in the Sun. We include background estimates. We then estimate the rate and sensitivity for current or proposed telescopes such as AMANDA-II, ANTARES, NESTOR and IceCube as well as for a $\mathrm{km}^{3}$ volume detector in the Mediterranean which would be equivalent to an expanded version of ANTARES and NESTOR.

\section{TAU DETECTED RATE FROM SUPERHEAVY DARK MATTER}

The neutrino flux spectrum from simpzilla annihilation in the Sun arriving at the Earth is given by [2]

$$
\frac{d F}{d E}=\frac{1}{4 \pi D^{2}}\left(\frac{d f}{d E}\right)_{\text {emergent }}=3.5 \times 10^{-18}\left(\frac{d f}{d E}\right)_{\text {emergent }} \mathrm{km}^{-2}
$$

where $D$ is the Sun-Earth distance and the differential in the right is the neutrino flux emerging from the Sun given in [2]. This flux depends on the simpzilla-nucleon cross section and on the simpzilla mass. Figure 4 shows the tau neutrino differential rate weighted by neutrino energy deduced from this equation.

This rate has to be weighted by the probability of conversion inside the detector. This probability is approximately $n \sigma_{C C}(E) L$ where $n$ is the target number density (ice or water for neutrino telescopes), $\sigma_{C C}(E)$ is the neutrino-nucleon charged current (CC) deep inelastic scaterring cross section and $L$ is the length of the detector assumed to be $1 \mathrm{~km}$. We determine $\sigma_{C C}(E)$ using CTEQ4-DIS parton distribution functions as described in [12]. Although the attenuation of the flux when going through the Earth is not significant we take it into account for completeness. This attenuation reduces the initial flux by $\exp \left[-\int n_{\oplus}\left(\sigma_{C C}+\sigma_{N C}\right) d x\right]$ where $x$ is the distance the neutrino travels through Earth, $n_{\oplus}$ is the Earth number density and $\sigma_{N C}$ is the neutrino-nucleon neutral current (NC) cross section. The event rate spectrum is given by

$$
\frac{d R}{d E}=\frac{d F}{d E}\left[n \sigma_{C C}(E) L\right] A \exp \left(-\int n_{\oplus}\left(\sigma_{C C}+\sigma_{N C}\right) d x\right)
$$

where $A$ is the area of the detector, assumed to be $1 \mathrm{~km}^{2}$. It is important to note that the detected rate only includes the contained events. We approximate the contained rate by including events where the neutrino converts into a lepton inside the geometrical detector volume.

A $\nu_{\tau}$ CC interaction with a nucleus will produce a $\tau$ lepton with energy $(1-y) E_{\nu}$ and a hadronic shower with energy $y E_{\nu}$, where $y$ is the fraction of energy transferred to the hadronic shower given in [26]. Once the tau is produced inside the detector it will almost immediately decay and produce another $\nu_{\tau}$ and another shower (hadronic or electromagnetic). The distance it travels inside the detector is an important characteristic that can be used at high energy for lepton identification. This will be discussed in the next section when we describe the double-bang technique [27].

As the tau production implies a simultaneous hadronic shower production and its decay might also generate another shower inside the detector its signature is different from one produced by a muon neutrino. The background for taus will include all interactions that produce a shower inside the detector. Also, as taus decay almost immediately, they have to be generated inside the detector. As muons travel a long way before decaying they can be produced outside the detector and yet be detected. Another important difference is that the detector angular resolution for a single particle as a muon is much better than for showers. Neutrino telescopes have angular resolution of about $1^{\circ}$ [28] for a long single-particle track and $30^{\circ}$ for a shower [21]. The backgrounds for tau events will be discussed in the next section as well as ways to reduce them.

In Fig. 5 we show the estimated event rate from simpzilla annihilation in the Sun. The rate is for events contained in a $\mathrm{km}^{3}$ volume and includes both taus and anti-taus [29]. The number of showers generated by a tau neutrino conversion into a tau versus the shower energy is shown. Since the tau will most likely decay very close to the vertex where the shower was produced, we consider that $100 \%$ of the tau neutrino energy went into the shower. Also shown are the largest backgrounds, i.e., assuming there is no angular resolution and taking the background from full sky. The background sources will be discussed in the next section but as can be seen in this figure-even in the worst case-the event rate is large and readily detected.

\section{A. Background}

The backgrounds for tau neutrino signature from simpzilla annihilation in the Sun are produced by any interaction 
that generates a shower inside the detector. Showers associated with muon events are not considered because the long muon track should be distinguishable from the shower. Three channels are considered: CC interaction $\nu_{e}+n \rightarrow e+X$ and NC interactions $\nu_{e}+n \rightarrow \nu_{e}+X$ and $\nu_{\mu}+n \rightarrow \nu_{\mu}+X$, where $n$ stands for nucleon. Sources of background neutrinos are produced either in the Earth or Sun atmospheres. The atmospheric flux of neutrinos is taken from [30]. An analysis of the difference between this spectrum and others can be found in [13]. The solar flux of neutrinos is taken from [31].

The average energy loss in both $\mathrm{CC}$ and $\mathrm{NC}$ interactions is $y=\left(1-E_{\text {lep }} / E_{\nu}\right)$ where $E_{\text {lep }}$ and $E_{\nu}$ are the lepton and neutrino energy, respectively. For neutrino energies between 10 and $100 \mathrm{GeV} y$ is about 0.48 gradually decreasing to about 0.2 at high energies [26]. At high energies the lepton gets about $80 \%$ of the neutrino energy. In CC interactions, the shower energy is the sum of all shower products, or $E_{\nu}$. In NC interactions, the shower energy is $E_{X}=y E_{\nu}$.

In Fig. 5 we show the background rates compared to the simpzilla signal. The differential shower rate weighted by shower energy is shown. We assume no angular resolution and no background reduction. Even in this worst possible scenario the signal can be detected for a large fraction of the simpzilla parameter space.

Background reduction will be useful for detecting simpzilla scenarios with low fluxes. At very high energy, tau neutrinos can be identified using the double bang signature [27] and the zenith angle dependence [32]. The double bang signature looks for two showers inside the detector. The tau will travel a distance $l_{\tau}$ given by

$$
l_{\tau}=\frac{E_{\tau} c t_{0}}{m_{\tau}}=\frac{(1-y) E_{\nu_{\tau}} c t_{0}}{m_{\tau}}
$$

where $E_{\tau}$ and $m_{\tau}$ are the tau energy and mass, $E_{\nu_{\tau}}$ is the tau neutrino energy, $c$ is the speed velocity and $t_{0}$ is the tau rest lifetime. If both showers occur inside the detector this double bang signature can distinguish the tau shower from other kind of showers. However, for this technique to work not only do the showers have to be sufficiently energetic and separated to be detected individually but also they have to be contained in the detector. At $100 \mathrm{TeV}$, the double bang is only $5 \mathrm{~m}$ separated and the two showers will be merged because in ice the scattering length of light is about $25 \mathrm{~m}$, and in water it is about $100 \mathrm{~m}$.

The second technique [32] is related to the spectral zenith angle dependence. Above an energy of $1 \mathrm{PeV}$ the Earth becomes opaque to electron and muon neutrinos. The tau regenerates, i.e., the tau produced in a neutrino $\mathrm{CC}$ interaction will almost immediately decay back into a tau neutrino. Therefore the tau neutrino will have its energy degraded when going through the Earth but at high energies will not have its flux attenuated. The electron or muon produced in the neutrino CC interaction will be absorbed and the flux will be attenuated. The tau neutrino flux will have its energy degraded to the energy with which its interaction length is of the order of the diameter of the Earth (which we call transparency energy). In this way the tau neutrino spectrum will be a pile up of events around the transparency energy

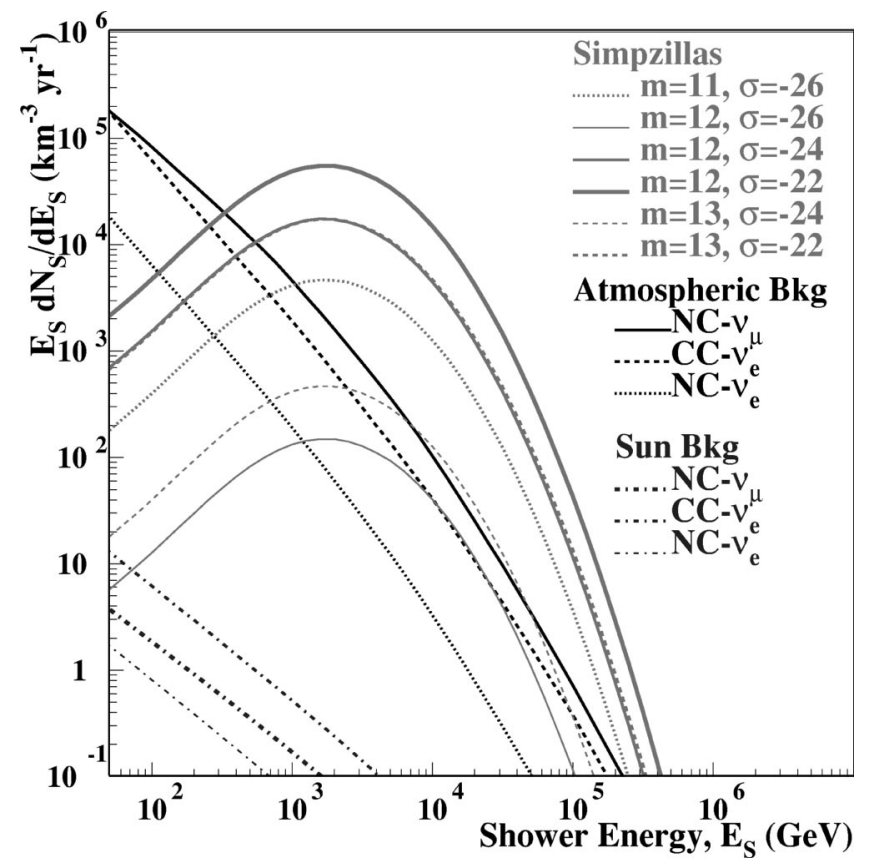

FIG. 5. Differential shower rate weighted by shower energy. Showers are produced from tau neutrino charged current interactions. The tau neutrinos are secondary products of simpzilla annihilation in the Sun. Different values for $M_{\chi}$ and $\sigma_{\chi-N}$ are assumed and labeled as $10^{\mathrm{m}} \mathrm{GeV}$ and $10^{\sigma}$. Also shown is the background of showers generated by muon neutrino-nucleon neutral current interactions, electron neutrino-nucleon charged and neutral current interactions from the Earth [30] and from the Sun [31]. The background is shown without any technique to be reduced. Even in this worse possible scenario the signal can be detected.

$(\sim 100 \mathrm{TeV})$. Using these two characteristics [32] the tau neutrino can be distinguished from the background by the pile up around $100 \mathrm{TeV}$. Also it will not have a zenith angle dependence since the flux will not be degraded. As the electron and muon neutrino have a strong zenith angle dependence [32] one can use the flat tau neutrino spectrum as a signature. It has also been proposed [33] that tau neutrinos above $\sim 100 \mathrm{TeV}$ will have their signature enhanced by the presence of anti-muon neutrinos and by anti-electron neutrinos.

The simpzilla spectrum is softer than $100 \mathrm{TeV}$, so background reduction in this analysis must come from the shower properties. Backgrounds will quickly dominate below $1 \mathrm{TeV}$, if energy cuts are not possible. The IceCube energy threshold for showers is $1 \mathrm{TeV}$ [21]. This threshold can be raised as needed to optimize the energy resolution. Some of the interesting models have many tens of thousands of events above this threshold.

Dimmer simpzilla models will be accessible using angular constraints. Energetic showers are boosted along the production direction. Charged particles in the shower produce Cherenkov light at broader angles. This light is also scattered as it travels to the PMTs. Preliminary studies indicate that reconstructed shower directions have an RMS angular resolution of $30^{\circ}$ [21]. Figure 6 shows the reduction of the background by a $30^{\circ}$ angular cut. One can see that this reduction will 


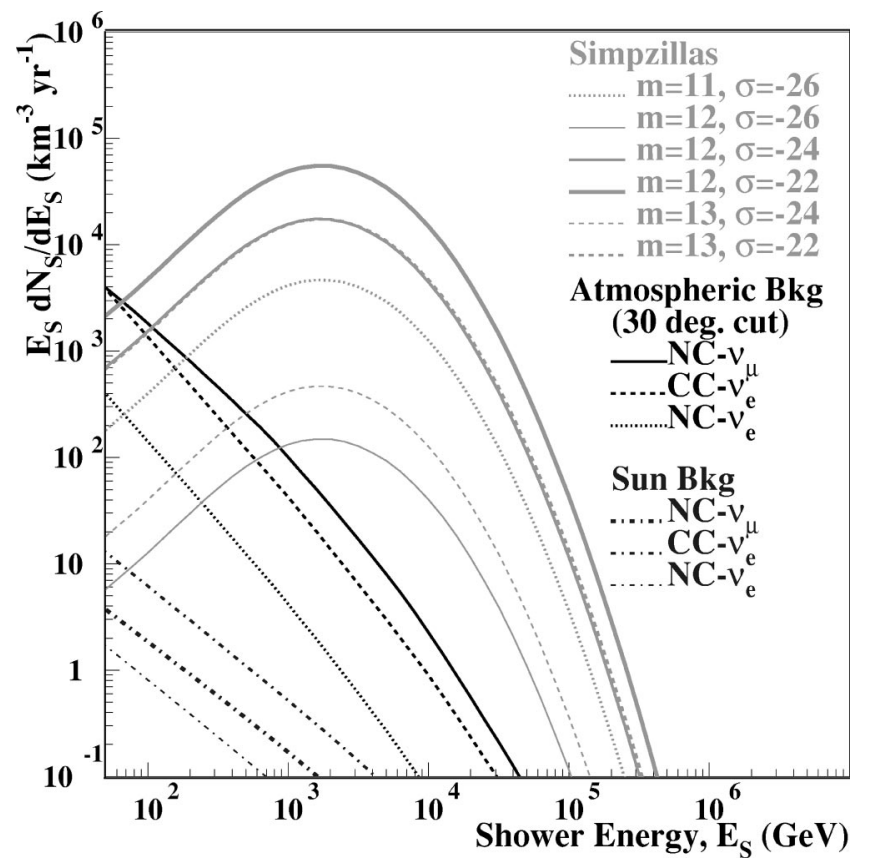

FIG. 6. Same as Fig. 5 but now with a $30^{\circ}$ angular resolution used to reduce the atmospheric background.

allow a broader simpzilla parameter space to be probed.

\section{B. Rate of current and proposed detectors}

As mentioned in the preceding section, the atmospheric background for simpzillas can be reduced using a $30^{\circ}$ angular cut. The background from the Sun is irreducible but yet very low when compared to the simpzilla signal (see Fig. 6).

Although neutrino telescopes have poor energy resolution it is possible to set a lower energy threshold. To optimize the energy threshold of detectors with the goal of probing up to a confidence level (C.L.) a broader simpzilla parameter space, we scale the signal to the background level for a fix set of Simpzilla parameters. This allows determination of flux limits and experiments sensitivity as described in Feldman and Cousins (FC) [34] and in [35]. In order to determine the optimum energy threshold the systematic uncertainty in the background for each detector has to be understood. This was estimated in [13] and results in a $25 \%$ background systematic error for a $\mathrm{km}^{3}$ detector and $50 \%$ for the smaller experiments as AMANDA-II, ANTARES and NESTOR. The determination of the experiments sensitivity using the FC approach is described in [13]. Our results are shown within 95\% C.L. Table I shows the optimum energy threshold for the different experiments. The IceCube energy threshold applies also for an expanded version of ANTARES or NESTOR. The optimum energy threshold optimizes the background/signal ratio in order to be able to probe a larger simpzilla parameter space region.

The signal and background estimated rate for the smaller experiments is found by reducing the rate by the same factor as for the volume reduction. The AMANDA-II detector has a geometrical volume of about $16 \times 10^{-3} \mathrm{~km}^{3}$ [28], ANTARES [19] of about $3 \times 10^{-2} \mathrm{~km}^{3}$ and NESTOR [18] of about $9 \times 10^{-3} \mathrm{~km}^{-3}$. It has been shown [13] that for these telescopes the geometrical efficiency as a function of the zenith angle varies between $100 \%$ to $90 \%$ for muons with a path length longer than $100 \mathrm{~m}$. This will be slightly different for showers but we will assume $100 \%$ geometrical efficiency for AMANDA-II, ANTARES and NESTOR detectors.

Figure 7 shows the Simpzilla parameter space to be probed by a neutrino telescope with $\mathrm{km}^{3}$ volume either in the South Pole (IceCube) or in the Mediterranean (an expanded version of ANTARES or NESTOR). The region excluded (hatched) is based on several experimental measurements [36] and the "low rate" region indicates where the neutrino flux is too low [2]. The lines show the region to be probed (above the line) within $95 \%$ C.L. The solid line is for a $\mathrm{km}^{3}$ experiment with no background reduction and the dotted line, including a $30^{\circ}$ angular cut, is to reduce the background. The smaller experiments must use a technique to reduce the background otherwise they cannot probe a significant simpzilla parameter region. The dashed line shows the region to be probed by ANTARES assuming a $30^{\circ}$ angular cut. Among the smaller experiments it can probe the largest parameter space. AMANDA and NESTOR will be similar and their limits are between the ANTARES and full sky $\mathrm{km}^{3}$ detector limits. This figure also shows the parameter space (dashed-dotted) for a $5 \sigma$ detection which assures discovery if simpzillas are the dark matter. This limit is for a $\mathrm{km}^{3}$ volume assuming a $30^{\circ}$ angular cut. ANTARES discovery limit is close to the $\mathrm{km}^{3}$ full sky limit.

In the most natural scenarios the simpzilla mass is close to the inflaton mass $\left(10^{12} \mathrm{GeV}\right)$ and the simpzilla-nucleon cross section is close to the nucleon-nucleon strong interaction cross section. As can be seen from Figs. 6 and 7 these

TABLE I. Optimum energy threshold (in TeV) for simpzilla detection. The results considering background from full sky and using a $30^{\circ}$ angular cut are shown.

\begin{tabular}{lcccc}
\hline \hline & \multicolumn{3}{c}{$95 \%$ C.L. } \\
\hline & $\begin{array}{c}\text { IceCube } \\
\left(\mathrm{km}^{3}\right)\end{array}$ & ANTARES & AMANDA-II & NESTOR \\
Full sky threshold $(\mathrm{TeV})$ & 20.0 & 6.3 & 4.0 & 3.2 \\
$30^{\circ}$ sky $(\mathrm{TeV})$ & 2.5 & 0.5 & 0.3 & 0.25 \\
& & $5 \sigma$ & 0.16 & 0.10 \\
$30^{\circ}$ sky $(\mathrm{TeV})$ & 1.3 & 0.25 & & \multirow{2}{*}{0.10} \\
\hline \hline
\end{tabular}


can be easily probed by neutrino telescopes of $\mathrm{km}^{3}$ volume. Also the smaller neutrino telescopes can probe a large region of the simpzilla parameter space.

\section{Neutrino oscillations}

There is strong evidence that neutrinos oscillate from one flavor to another. Superkamiokande [38] determines the most probable solution for the difference between the squared masses of the two neutrino mass eigenstates $\left(\Delta \mathrm{m}^{2}\right)$ as 3.5 $\times 10^{-3} \mathrm{eV}^{2}$ and a large mixing angle for atmospheric neutrinos. It also favors muon neutrino oscillation into tau neutrinos [39]. SNO [40] has recently determined $\Delta m^{2}$ to be $5.0 \times 10^{-5} \mathrm{eV}^{2}$ in the large mixing angle scenario for solar neutrinos. Atmospheric neutrino oscillations will be important only below $\sim 200 \mathrm{GeV}$ [41]. This is below the energy threshold for the simpzilla analysis and therefore has no effect in the background considered here. However, neutrino oscillations might occur in the Sun and in transit to Earth and therefore affect the simpzilla signature. One should note that the neutrinos from simpzilla annihilation have energies much higher than the standard solar neutrinos. Oscillation of three neutrino flavors are analyzed in the GeV energy regime [42]. One important result is that for some regions of the oscillation parameter space (which now includes three mixing angles) it is possible to have an enhancement of tau neutrinos detected in the Earth (when coming from the Sun) with respect to the number of muon neutrinos (or vice versa).

Crotty [43] analyzes the effect of oscillations in the neutrino flux from simpzilla annihilation in the Sun assuming fixed values for the three flavor oscillation parameters. $\Delta m_{31}^{2}$ is taken as $3 \times 10^{-3} \mathrm{eV}^{2}, \sin ^{2} \xi=0.1, \sin ^{2} \theta=0.5, \Delta m_{21}^{2}=2$ $\times 10^{-5} \mathrm{eV}^{2}$ and $\sin ^{2} \omega=0.2$. It is also assumed the "normal" mass hierachy where $m_{1}<m_{2}<m_{3}$. See [43] for a description of these parameters. The result is that the tau neutrino estimated rate at the Earth and the tau neutrino contained rate in a $\mathrm{km}^{3}$ volume will be reduced while the muon neutrino event rate will be enhanced. It has also been shown [33] that secondary muon neutrinos (see Sec. III) will enhance the muon neutrino flux by about $20 \%$ of the tau neutrino flux at the Earth. Taking both secondaries and oscillation into account, the tau neutrino and muon neutrino flux at the Earth become approximately equal [43]. The conversion rate in a $\mathrm{km}^{3}$ volume will also be approximately equal (with a slightly higher muon neutrino rate) [43]. The tau neutrino detected rate will then be approximately half of that without oscillations.

This reduction in the tau neutrino rate does not reduce the simpzilla parameter space to be probed by neutrino telescopes which are also able to detect muon neutrinos. The region to be probed by tau neutrino detection would be reduced. The $\mathrm{km}^{3}$ with $30^{\circ}$ sky limit in Fig. 7 would be moved to where the 5 sigma limit stands without oscillation. The ANTARES limit will move very close to the $\mathrm{km}^{3}$ full sky limit. The limit that will be most affected will be the $5 \sigma$ which will also move close to the $\mathrm{km}^{3}$ full sky limit. However, once oscillations are considered there will be a muon neutrino flux at the same level of the tau neutrino flux. As mentioned before (see Sec. IV) the neutrino telescopes angu-

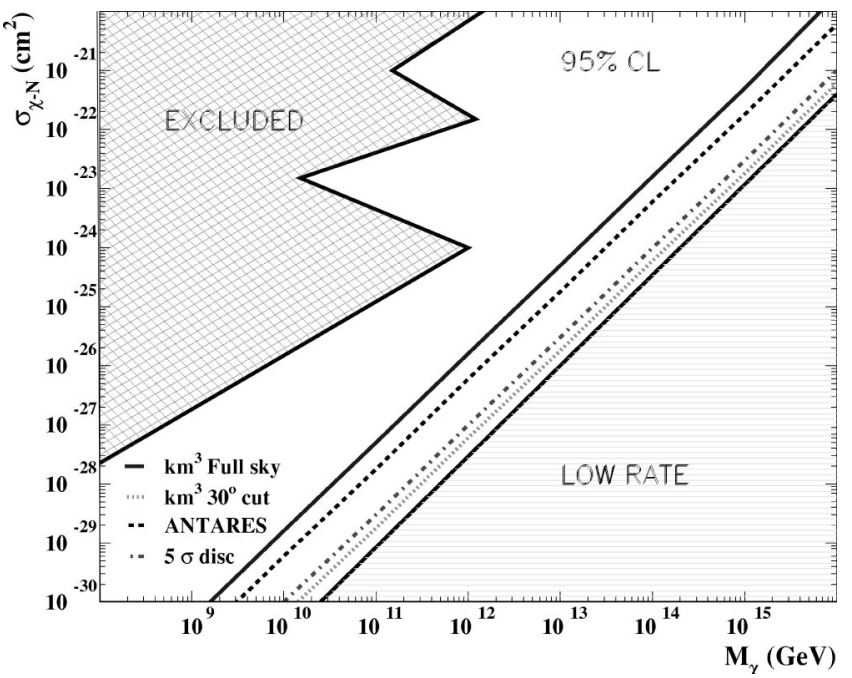

FIG. 7. The clear region below the jagged line shows the allowed simpzilla $M_{\chi}$ versus $\sigma_{\chi-N}$ space. The hatched region above the jagged line is excluded by several arguments discussed in [36]. More model dependent constraints can be found in [36,37]. The lined region indicates where the neutrino flux is too low [2]. The lines show the region to be probed with $95 \%$ C.L. (above the line) in one year of full operation. The solid line is for a $\mathrm{km}^{3}$ experiment (such as IceCube) with no background reduction and the dotted line including a $30^{\circ}$ angular cut to reduce the background. The dashed line shows the region to be probed by ANTARES assuming a $30^{\circ}$ angular cut. AMANDA-II and NESTOR limits are between the ANTARES limit and the full sky IceCube limit. The limits from the smaller telescopes include a $30^{\circ}$ angular cut to reduce the background. The dashed-dotted line is the $\mathrm{km}^{3}$ volume $5 \sigma$ limit. The limits for $\mathrm{a} \mathrm{km}^{3}$ volume detector assumes that the detector is either in the South Pole (IceCube) or in the Mediterranean Sea (an expanded version of ANTARES or NESTOR).

lar resolution is much better for muons (about $1^{\circ}$ ) than for the tau generated showers. This will reduce the background to a much lower level than for the tau neutrino analysis. As a result the Simpzilla parameter space to be probed is the same as without oscillations or even larger. This is the subject of future work.

\section{CONCLUSION}

We have discussed the indirect detectability of dark matter by neutrino telescopes. The decay of secondary products of dark matter annihilation would generate a flux of high energy neutrinos.

We have argued that when current and proposed neutrino telescopes' energy threshold and location are taken into account the detection of the neutralino as a WIMP is borderline. Most of the WIMP parameter space is inaccessible even in tens of years of observation. The parameter space which can be probed in 5 or more years of $100 \%$ live time can also be probed by direct detection, making the techniques complementary.

However if the dark matter is constituted by superheavy (mass $\geq 10^{10} \mathrm{GeV}$ ) dark matter (Simpzillas), most of the current and proposed neutrino telescopes can probe the most 
natural scenarios. In the most natural scenario, the simpzilla mass is similar to the inflaton mass $\left(10^{12} \mathrm{GeV}\right)$ and the simpzilla-nucleon cross section is the same as the strong interaction cross section $\left(10^{-26} \mathrm{~cm}^{2}\right)$.

Proposed detectors such as IceCube or an expanded version of ANTARES or NESTOR are able to probe Simpzillas to $5 \sigma$ having a significant signal rate. Figure 7 shows the simpzilla mass versus simpzilla-nucleon cross-section region to be probed. A $30^{\circ}$ angular cut efficiently reduces the background without any loss in the signal rate. This cut allows most of the currently allowed simpzilla parameter space to be probed.

If oscillations are taken into account tau neutrinos might convert into muon neutrinos. Crotty [43] shows that under certain parameter assumptions this might reduce the tau neutrino detected rate by about a factor of two and enhance the muon neutrino detected rate. As these muon neutrinos can be detected, not only the simpzilla parameter space to be probed will continue to be as shown in Fig. 7 but it might even allow for neutrino oscillation studies.
The smaller neutrino telescopes, AMANDA-II and current versions of ANTARES and NESTOR, are able to probe a large fraction of simpzilla mass versus simpzilla-nucleon cross-section parameter space.

As a final conclusion, although WIMPs indirect detection of most models will take tens of years, current and proposed neutrino telescopes can either discover or rule out the most natural scenarios for superheavy strongly interacting massive particles in one year of full operation.

\section{ACKNOWLEDGMENTS}

We thank Joakim Edsjo and Willi Chinowsky for comments. This work was supported by NSF Grants KDI 9872979 and Physics/Polar Programs 0071886 and in part by the Director, Office of Energy Research, Office of High Energy and Nuclear Physics, Division of High Energy Physics of the U.S. Department of Energy under Contract No. DEAC03-76SF00098 through the Lawrence Berkeley National Laboratory.
[1] G. Jungman, M. Kamionkowski, and K. Griest, Phys. Rep. 267, 195 (1996).

[2] I.F.M. Albuquerque, L. Hui, and E.W. Kolb, Phys. Rev. D 64, 083504 (2001)

[3] R. Abusaidi et al., Phys. Rev. Lett. 84, 5699 (2000).

[4] L. Baudis et al., astro-ph/0005568.

[5] J. Edsjo, Nucl. Phys. B43, 265 (1997).

[6] L. Bergstrom, J. Edsjo, and M. Kamionkowski, Astropart. Phys. 7, 147 (1997).

[7] L. Bergstrom, J. Edsjo, and P. Gondolo, Phys. Rev. D 55, 1765 (1997).

[8] L. Bergstrom, J. Edsjo, and P. Gondolo, Phys. Rev. D 58, 103519 (1998).

[9] G. Jungman and M. Kamionkowski, Phys. Rev. D 51, 328 (1995).

[10] A. Gould, Astrophys. J. 321, 571 (1987).

[11] W.H. Press and D. Spergel, Nucl. Phys. B283, 681 (1987).

[12] R. Gandhi, C. Quigg, M.H. Reno, and I. Sarcevic, Phys. Rev. D 58, 093009 (1998)

[13] I.F.M. Albuquerque, J. Lamoureux, and G.F. Smoot, Astrophys. J., Suppl. Ser. 141, 195 (2002).

[14] M. Battaglia et al., Eur. Phys. J. C 22, 535 (2001).

[15] J. Ellis, J.L. Feng, A. Ferstl, K.T. Matchev, and K. Olive, Eur. Phys. J. C 24, 311 (2002).

[16] H.P. Nilles, Phys. Rep. 110, 1 (1984).

[17] E. Andres et al., astro-ph/9906205.

[18] L.K. Resvanis, Nucl. Phys. B (Proc. Suppl.) 87, 448 (2000). Talk given at the 8th International Workshop on Neutrino Telescopes, Venice, Italy, 1999.

[19] J. R. Hubbard et al. and L. Moscoso et al., in Proceedings of the 26th International Cosmic Ray Conference, Salt Lake City, Utah, 1999.

[20] M. Leuthold, presented at the International Workshop on Simulations and Analysis Methods for Large Neutrino Telescopes, Zeuthen, Germany, 1998.
[21] J. Ahrens et al., "IceCube Conceptual Design Document," http://icecube.wisc.edu/tech/, 2001.

[22] J. Ahrens et al., Phys. Rev. D 66, 012005 (2002).

[23] J. Ahrens et al., Phys. Rev. D 66, 032006 (2002).

[24] V. Bertin, E. Nezri, and J. Orloff, hep-ph/0204135.

[25] D.J.H. Chung, E.W. Kolb, and A. Riotto, Phys. Rev. Lett. 81, 4048 (1998); Phys. Rev. D 59, 023501 (1998); 60, 063504 (1999).

[26] R. Gandhi, C. Quigg, M.H. Reno, and I. Sarcevic, Astropart. Phys. 5, 81 (1996).

[27] J.G. Learned and S. Pakvasa, Astropart. Phys. 3, 267 (1995).

[28] S. Barwick, in Proceedings of the 27th International Cosmic Ray Conference, Copernicus Gesellschaft, 2001.

[29] From now on when taus are mentioned they include the antitaus.

[30] L.V. Volkova, Yad. Fiz. 31, 1510 (1980). Also published at Sov. J. Nucl. Phys. 31, 784 (1980).

[31] G. Ingelman and M. Thunman, Phys. Rev. D 54, 4385 (1996).

[32] F. Halzen and D. Saltzberg, Phys. Rev. Lett. 81, 4305 (1998).

[33] J.F. Beacom, P. Crotty, and E.W. Kolb, Phys. Rev. D 66, 021302 (2001).

[34] G.J. Feldman and R.D. Cousins, Phys. Rev. D 57, 3873 (1998).

[35] D.E. Groom et al., Eur. Phys. J. B 15, 195 (2000).

[36] G.D. Starkman, A. Gould, R. Esmailzadeh, and S. Dimopoulos, Phys. Rev. D 41, 3594 (1990).

[37] C. Bacci, et al., Astropart. Phys. 2, 13 (1994); R. Bernabei et al., Phys. Rev. Lett. 83, 4918 (1999).

[38] Y. Fukuda et al., Phys. Rev. Lett. 85, 3999 (1998).

[39] Y. Fukuda et al., Phys. Rev. Lett. 81, 1562 (2000).

[40] Q.R. Ahmad et al., Phys. Rev. Lett. 89, 011302 (2002).

[41] I.F.M. Albuquerque and G.F. Smoot, Phys. Rev. D 64, 053008 (2001).

[42] A. de Gouvêa, Phys. Rev. D 63, 093003 (2001).

[43] P. Crotty, Phys. Rev. D 66, 063504 (2002); Ph.D. dissertation. Department of Physics, The University of Chicago, 2002. 